\title{
Nucleoside Analogues as Antibacterial Agents
}

\author{
Jessica M. Thomson and lain L. Lamont* \\ Department of Biochemistry, University of Otago, Dunedin, New Zealand
}

The rapid increase in antibiotic-resistant bacteria has emphasized the urgent need to identify new treatments for bacterial infections. One attractive approach, reducing the need for expensive and time-consuming clinical trials, is to repurpose existing clinically approved compounds for use as antibacterial agents. Nucleoside analogues are commonly used for treating viral and fungal infections, as well as for treating cancers, but have received relatively little attention as treatments for bacterial infections. However, a significant number of clinically approved derivatives of both pyrimidines and purines including halogenated, thiolated, and azolated compounds have been shown to have antibacterial activity. In the small number of studies carried out to date, such compounds have shown

OPEN ACCESS

Edited by:

Rustam Aminov,

University of Aberdeen,

United Kingdom

Reviewed by:

Babita Agrawal,

University of Alberta, Canada

Steven W. Polyak,

University of South Australia,

Australia

${ }^{*}$ Correspondence:

lain L. Lamont

iain.lamont@otago.ac.nz

Specialty section:

This article was submitted to Antimicrobials, Resistance and

Chemotherapy,

a section of the journal

Frontiers in Microbiology

Received: 29 January 2019

Accepted: 15 April 2019

Published: 22 May 2019

Citation:

Thomson JM and Lamont IL (2019)

Nucleoside Analogues as Antibacterial Agents.

Front. Microbiol. 10:952.

doi: 10.3389/fmicb.2019.00952 promise in treating bacterial infections. Here, we review the mechanisms of action and antibacterial activities of nucleoside analogues that can potentially be repurposed for treating infections as well as considering possible limitations in their usage.

Keywords: repurposed antibiotics, antibiotic resistance, pyrimidine analogues, purine analogues, multidrug-resistant bacteria, antibacterial agents, antimicrobial

\section{INTRODUCTION}

The introduction of antibiotics into clinical use heralded a new age for medicine. However, less than a century later, the therapeutic efficacy of antibiotics is becoming limited owing to the rise of resistance in pathogenic bacteria (Lewis, 2013). Once a problem largely limited to hospital environments, antibiotic-resistant strains of pathogens have progressively become more prevalent in the community, and their spread has been unrestricted (Rice, 2009). It has been estimated that there are at least 700,000 deaths a year worldwide due to infections by antibioticresistant bacteria and in the absence of new treatment strategies this figure could rise to 10 million deaths a year by 2050 (O'Neill, 2014). Despite an urgent need for new antibiotics to combat resistant pathogens, there have been very few novel antibiotics to make it into clinical practice. Indeed, the majority of antibiotics in use today belong to classes discovered before the 1970s (Lewis, 2013). There are a number of factors contributing to the issue of failing antibiotic discovery, including declining interest in discovery by pharmaceutical companies due to the high costs of bringing drugs to market and limited approaches to identify lead compounds (Livermore, 2011). However, some promising approaches to antibiotic discovery do exist. Recently, novel methods of screening environmental microbes have yielded promising antibiotic compounds (Gavrish et al., 2014; Ling et al., 2015). While such screening techniques hold promise for discovery of novel compounds, it will be a number of years before any discovered compounds are sufficiently studied to be used clinically. Thus, a different approach to discovery, namely drug repurposing, may identify compounds with antibiotic activity and 
represents a way to fast track them into clinical use (Brown, 2015; Rangel-Vega et al., 2015; Miro-Canturri et al., 2019). The concept behind repurposing is that almost all drugs in clinical use exhibit various pharmacological activities secondary to their main activity. Screening these compounds for side activities, and optimizing activity if required, could represent a way to identify new antibiotics. As the safety profiles of the drugs are known, clinical trials could be less extensive, thus reducing costs associated with approval and allowing drugs to be used clinically more rapidly than completely novel drugs.

One class of drugs that are important from a clinical perspective is nucleoside analogues, a pharmacologically diverse class of drugs that arose from chemically modified natural ribose or 2'-deoxyribose nucleosides (Koszytkowska-Stawinska and Buchowicz, 2014). Nucleoside analogues are among the most important drugs in the clinical setting and are used widely as both anticancer and antiviral agents (Jordheim et al., 2013). Nucleoside analogues mimic endogenous nucleosides, exploiting cellular metabolism and becoming incorporated into both DNA and RNA. This property makes nucleoside analogues effective at inhibiting viral replication and stopping cancer cell proliferation. However, although there have been some studies on the efficacy of nucleoside analogues as antibacterial agents, the potential use of these compounds in treating bacterial infections has been relatively neglected.

For the purposes of this review, a nucleoside analogue is defined as a nucleobase linked to a sugar, where the nucleobase or the sugar component is altered such that the molecule becomes distinct from those found naturally. Modifications to nucleobases include halogenation and addition of azido groups, and modifications of the sugar component include ring opening, halogenation, methylation, and hydroxylation or dehydroxylation (Jordheim et al., 2013). It is worth noting that a class of drugs called nucleoside antibiotics has also been studied for potential clinical use as antibacterials. Nucleoside antibiotics typically consist of parts of natural nucleosides conjugated with additional complex structures such as amino acids or fatty acids, with the combination having antibacterial properties. In bacteria, nucleoside antibiotics primarily target cell-wall biosynthesis (Winn et al., 2010; Niu and Tan, 2015). The potential use of various nucleoside antibiotics has been extensively reviewed elsewhere (Winn et al., 2010; Carter and McDonald, 2014; Niu and Tan, 2015) and is beyond the scope of this review.

This review will cover nucleoside analogues that have shown clinical potential for repurposing as antibacterials. A summary of the metabolism of the relevant compounds is shown in Figure 1. First, their usage and modes of action in treating cancer or viral infections, along with studies validating their antibiotic activity against bacteria, will be outlined. Second, studies on the metabolism of nucleoside analogues and their mechanism of action in bacteria will be discussed. Finally, limitations of nucleoside analogues as antibiotics including potential for toxicity and development of resistance will be considered.

Abbreviations: dNK, Deoxyribonucleoside kinase; HPRT, Hypoxanthine-guanine phosphoribosyl transferase; TK, Thymidine kinase.

\section{PYRIMIDINE ANALOGUES}

\section{Gemcitabine}

Gemcitabine $\left(2^{\prime}, 2^{\prime}\right.$-difluoro-2'-deoxycytidine) is an analogue of deoxycytidine and has two fluorine atoms in place of hydrogen atoms on the $2^{\prime}$ carbon of the sugar component (Figure 2A). Gemcitabine was first developed as an antiviral drug but has since been used exclusively as an anticancer drug (Mini et al., 2006). Gemcitabine is used alone or in combination with other drugs for various types of cancers, including metastatic pancreatic cancer (Burris et al., 1997; Ishii et al., 2005). Gemcitabine is a prodrug, which is taken up into eukaryotic cells, phosphorylated to gemcitabine monophosphate by deoxycytidine kinase, and converted to the active metabolites gemcitabine di- and triphosphate (Heinemann et al., 1988; Mini et al., 2006). Once activated, gemcitabine is incorporated into growing DNA (Figure 1), ultimately resulting in termination of strand elongation. After incorporation of gemcitabine, the DNA polymerase adds one additional deoxynucleotide and DNA synthesis then ceases; the additional deoxynucleotide may mask gemcitabine from proof-reading exonucleases, preventing its removal (Huang and Plunkett, 1995). It is unclear why only one nucleotide is added after gemcitabine, but it may be that the drug induces DNA conformation changes, preventing the addition of further nucleotides (Plunkett et al., 1995). Gemcitabine can also potently inhibit ribonucleotide reductase (Figure 1; Huang and Plunkett, 1995). There is evidence that a metabolite of gemcitabine can be incorporated into RNA, although the identity of this metabolite and the impact this has on cells are unclear (Figure 1; Vanhaperen et al., 1993).

Gemcitabine has been the subject of various repurposing studies. In a study that explored the efficacy of various nucleoside analogues against clinical isolates from different bacteria genera, gemcitabine was identified as having antibacterial activity (Sandrini et al., 2007b). Gemcitabine had a potent antibacterial effect on Gram-positive bacteria, including important pathogenic species from genera Listeria, Bacillus, Enterococcus, and Staphylococcus, although it was ineffective against Gram-negative bacteria. A murine infection model was used to investigate whether gemcitabine was useful as an antibiotic in vivo (Sandrini et al., 2007a). Mice were infected with Streptococcus pyogenes AP1, a virulent strain responsible for causing the majority of severe $S$. pyogenes infections. Of the mice infected with a potentially fatal dose of $S$. pyogenes AP1, those treated with the control had a $100 \%$ mortality rate, whereas those treated with gemcitabine had only a $17 \%$ mortality rate. This demonstrated that gemcitabine had potent activity against S. pyogenes in vivo.

These studies showed gemcitabine to be a good candidate for repurposing as an antibiotic. Jordheim et al. (2012) performed additional in vitro preclinical studies to investigate the potential of gemcitabine, especially its efficacy against clinically important multidrug-resistant strains of Staphylococcus aureus. Gemcitabine was found to be active against 19 different strains of methicillinresistant $S$. aureus. Gemcitabine was effective against glycopeptideintermediate $S$. aureus, a strain resistant to all glycopeptide antibiotics, including vancomycin. Resistance to gemcitabine 

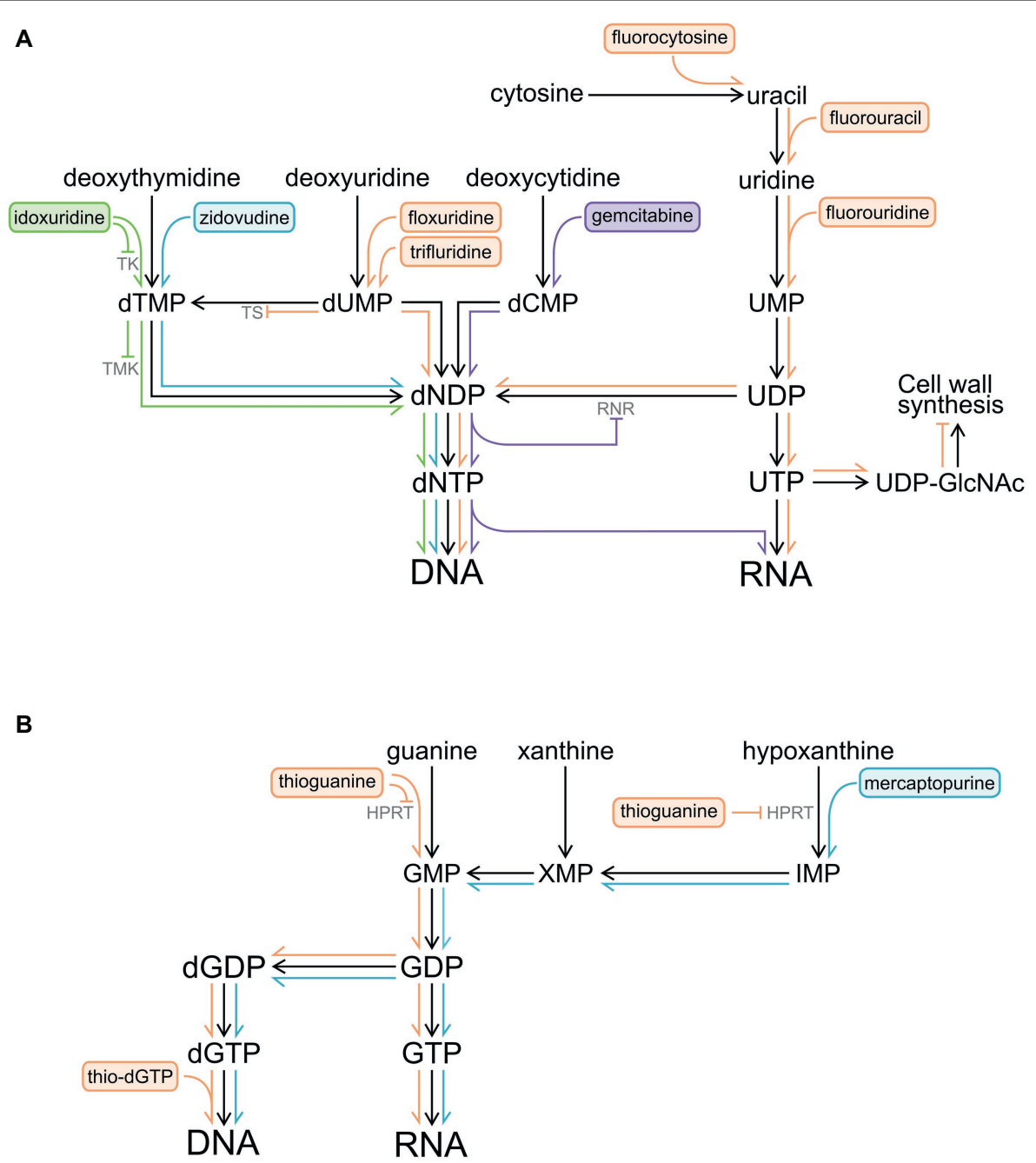

FIGURE 1 | Pathways by which pyrimidine and purine analogues are metabolized and become incorporated into DNA and RNA. Inhibition of enzymes by nucleoside analogues and their metabolites is also shown (-1). (A) Pyrimidine analogues. (B) Purine analogues. Abbreviations: HPRT, hypoxanthine-guanine phosphoribosyl transferase; IMP, inosine monophosphate; RNR, ribonucleotide reductase; TK, thymidylate kinase; TMK, deoxythymidine monophosphate kinase; TS, thymidylate synthase; UDP-GIcNAc, uridine diphosphate N-acetylglucosamine; XMP, xanthosine monophosphate.

could develop in treated S. aureus (Jordheim et al., 2012). However, gemcitabine was found to have synergistic activity with gentamicin, and if used in combination, emergence of resistance to these drugs may be slowed (Jordheim et al., 2012).

\section{Zidovudine}

Zidovudine [3'azido-3'-deoxythymidine, AZT] is a thymidine analogue with an azido group in place of the hydroxyl group at the $3^{\prime}$ carbon of the deoxyribose ring (Figure 2B). Zidovudine is an antiretroviral agent and is used clinically as therapy for HIV/AIDS. Once activated to its triphosphorylated form, zidovudine inhibits viral replication. The azido group of zidovudine prevents phosphodiester bond formation and results in DNA chain termination (Furman et al., 1986; Cooper and Lovett, 2011). Zidovudine is effective as an antiretroviral because it has an affinity for the viral reverse transcriptase that is approximately 100 -fold greater than its affinity for human DNA polymerase (Furman et al., 1986). However, zidovudine has still been found to be incorporated into the DNA of patients taking the drug, and there is evidence that at high doses, zidovudine can lead to various toxicities, including mitochondrial toxicity and cardiomyopathy (Lewis et al., 1992, 2000).

Zidovudine was first found to have antibacterial activity in the late 1980s. Zidovudine had antimicrobial activity against various Enterobacteriaceae, including Salmonella species (Elwell et al., 1987). Zidovudine was activated in these bacteria by thymidine kinase (TK), and incorporation of activated zidovudine into bacterial DNA resulted in DNA chain termination (Figure 1; Elwell et al., 1987). Subsequently, zidovudine has been demonstrated to have potent activity against many pathogenic Gram-negative bacteria, including Escherichia coli, Salmonella typhimurium, Klebsiella pneumoniae, Shigella flexneri, and Haemophilus influenzae and including isolates resistant to conventional antibiotics (Keith et al., 1989; Sandrini et al., 2007a,b; 
<smiles>Nc1ccn(C23OC(CO)(C(O)C2O)C(O)C3(O)F)c(=O)n1</smiles>

Deoxycytidine<smiles>Cc1cn(C2CC(O)C(CO)O2)c(=O)[nH]c1=O</smiles>

Thymidine<smiles>Cc1cn(C2CC(N=[N+]=[N-])C(CO)O2)c(=O)[nH]c1=O</smiles>

Zidovudine<smiles>O=c1[nH]c(=O)n(C2CC(O)C(CO)O2)cc1I</smiles>

Idoxuridine
C<smiles>Nc1cc[nH]c(=O)n1</smiles>

Cytosine<smiles>Nc1nc(=O)[nH]cc1F</smiles>

Fluorocytosine<smiles>O=c1cc[nH]c(=O)[nH]1</smiles>

Uracil

D<smiles>O=c1[nH]cc(F)c(=O)[nH]1</smiles>

Fluorouracil<smiles>O=c1[nH]c(=O)n(C2OC(CO)C(O)C2O)cc1F</smiles>

Fluorouridine<smiles>O=c1[nH]c(=O)n(C2CC(O)C(CO)O2)cc1F</smiles>

Floxuridine

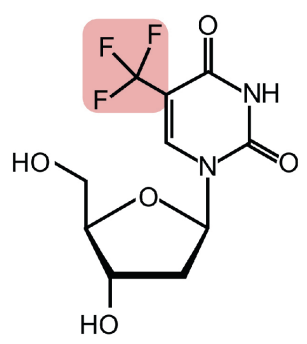

Trifluridine<smiles>O=c1[nH]cnc2[nH]cnc12</smiles>

Hypoxanthine<smiles>Nc1nc2[nH]cnc2c(=O)[nH]1</smiles>

Guanine<smiles>S=c1[nH]cnc2[nH]cnc12</smiles>

Mercaptopurine<smiles>Nc1nc2[nH]cnc2c(=S)[nH]1</smiles>

Thioguanine

FIGURE 2 | Structures of pyrimidines, purines, and their analogues, with substituted atoms highlighted. (A) Deoxycytidine and its analogue. (B) Thymidine and its analogues. (C) Cytosine, uracil, and fluorinated pyrimidines. (D) Hypoxanthine, guanine, and their analogues.

Doleans-Jordheim et al., 2011; Peyclit et al., 2018). It also acts synergistically with conventional antibiotics, enhancing their effectiveness (Wambaugh et al., 2017; Ng et al., 2018; Falagas et al., 2019; Hu et al., 2019). Zidovudine is ineffective against Gram-positive bacteria such as Listeria species, Bacillus species, Staphylococci, and Enterococcus faecalis as well as against Mycobacteria species and Pseudomonas aeruginosa (Elwell et al., 1987; Sandrini et al., 2007a).

The antibacterial activity of zidovudine has been demonstrated both in vitro and in vivo. Herrmann and Lagrange (1992) used a macrophage cell line to demonstrate that zidovudine inhibited intracellular growth of $S$. typhimurium. Zidovudine had potent in vivo activity. Zidovudine prevented lethal infections in mice with pyelonephritis caused by $E$. coli infection, being as effective as either trimethoprim or ampicillin (Keith et al., 1989). It also inhibited growth of antibiotic-resistant E. coli and K. pneumoniae in a murine peritoneal infection model, acting synergistically with colistin ( $\mathrm{Hu}$ et al., 2019). When administered subcutaneously, zidovudine also prevented lethal salmonellosis in calves infected with S. dublin (Keith et al., 1989). Zidovudine has therapeutic potential for humans as well; zidovudine given as an antiretroviral to HIV/AIDS patients also had the additional protective effect of lowering the recurrence of Salmonella bacteremia, a significant problem for HIV/AIDS patients (Casado et al., 1999). These in vivo findings suggest that zidovudine has potential application as an antibacterial agent. Zidovudine has also been the subject of modification studies, which aim to improve its therapeutic efficacy and resolve issues like short half-life of the drug. Research has gone into creating zidovudine derivatives that retain antiviral activity while having improved bactericidal activity (Moroni et al., 2002). Such derivatives may be particularly useful for HIV/AIDS patients; HIV/AIDS patients are susceptible to opportunistic bacterial infections, and improved bactericidal profile of these derivatives would be a beneficial side activity.

\section{Fluorinated Pyrimidines}

Originally synthesized as antitumor drugs (Heidelberger et al., 1957), fluorinated pyrimidines have also been used widely as antifungals (Vermes et al., 2003), have some use as antivirals (Wilhelmus, 2010), and show promise as antibacterials. The fluorinated pyrimidine family was first synthesized after the observation that tumor cells preferentially utilized uracil for nucleic acid biosynthesis (Rutman et al., 1954; Heidelberger et al., 1957). From this large family of compounds, the nucleobase 5-fluorouracil and the nucleoside floxuridine (5-fluoro$2^{\prime}$ deoxyuridine, Figure 2C) are frequently used for the treatment of various cancers (Galmarini et al., 2002; Alvarez et al., 2012). 
While these compounds are effective anticancer drugs and are taken up more rapidly by cancerous cells, they also affect non-cancerous cells, and their use is associated with a number of side effects, such as bone marrow depression (Galmarini et al., 2002). Trifluridine (Figure 2C) is used as an antiviral and is a therapy for herpetic simplex virus epithelial keratitis (Wilhelmus, 2010). 5-Fluorocytosine (Figure 2C), a prodrug of 5-fluorouracil, is used as an antifungal agent. 5-Fluorocytosine is most commonly used in combination therapy with other antifungals, typically amphotericin $\mathrm{B}$, as resistance to 5-fluorocytosine arises readily (Bennett, 1977; Francis and Walsh, 1992; Ghannoum and Rice, 1999). 5-Fluorocytosine itself is not active; antifungal activity is dependent upon uptake of 5-fluorocytosine into fungal cells and subsequent deamination to 5-fluorouracil (Figure 1; Bennett, 1977). In turn, 5-fluorouracil is converted to floxuridine mono-, di-, and tri-phosphate. Metabolites of 5-fluorouracil and floxuridine can become incorporated into DNA and RNA and can also inhibit thymidylate synthase, preventing de novo formation of deoxythymidine monophosphate (Figure 1; Bennett, 1977).

Compounds from the fluorinated pyrimidine family have antibacterial activity. Various fluorinated pyrimidines could inhibit the growth of the human pathogen Ureaplasma urealyticum (Carnrot et al., 2003). Floxuridine had a strong effect in vitro, inhibiting the growth of $U$. urealyticum for up to $72 \mathrm{~h}$. This finding was supported by a later study (Wehelie et al., 2004). Subsequently, various Gram-positive and Gram-negative bacteria were found to be susceptible to floxuridine (Sandrini et al., 2007a,b; Zander et al., 2010a). Gram-positive bacteria, such as various streptococci, S. aureus and Bacillus species, were more sensitive than the Gram-negative bacteria tested. Floxuridine has also been found to have synergistic effects when used in combination with zidovudine (Wambaugh et al., 2017). 5-Fluorouracil has been reported to decrease virulence of $P$. aeruginosa by disrupting biofilm formation (Ueda et al., 2009) and also has activity against Mycobacterium tuberculosis and a number of oral microbes including the pathogen S. pyogenes (Singh et al., 2015; Vanlancker et al., 2016). Floxuridine and trifluridine both affected the growth of Mycoplasma pneumoniae, with trifluridine strongly inhibiting growth in vitro (Sun and Wang, 2013). These studies highlight the antibacterial activity of fluorinated pyrimidines.

The possible application of fluorinated pyrimidines as antibacterials has been tested in vivo (Walz et al., 2010; Imperi et al., 2013; Kirienko et al., 2016). 5-Fluorouracil was demonstrated to be a clinically useful antibacterial agent by Walz et al. (2010). In a phase III clinical trial, Walz and colleagues studied the efficacy of central venous catheters coated with 5-fluorouracil in decreasing catheter-related bloodstream infections and reducing colonization of catheters by bacteria. Nearly 1,000 patients were randomized into two groups, one receiving a catheter coated with 5-fluorouracil and the other group receiving standard catheters coated with chlorhexidine-silver sulfadiazine (Walz et al., 2010). The catheters coated with 5-fluorouracil were as effective at preventing bacterial colonisation as the standard chlorhexidinesilver sulfadiazine catheters. The frequency of adverse events was comparable between the two groups. The dose of 5-fluorouracil used to coat the catheter was very low, and in a small tissue sample obtained from the location of catheterization, no toxicity was observed in patients receiving 5-fluorouracil-coated catheters (Walz et al., 2010). These data suggest that if widespread resistance to compounds currently impregnated in standard catheters were to arise, then 5-fluorouracil impregnated catheters could be clinically useful as an alternative. It is also encouraging that the 5-fluorouracil coating the catheters did not cause any toxicity or significant side effects.

While 5-fluorouracil has been shown to be a potentially effective antimicrobial, the drug does not specifically target bacteria and is also toxic to eukaryotic cells (Alvarez et al., 2012). Conversely, 5-fluorocytosine is a prodrug and is only converted to 5 -fluorouracil by susceptible cells. Human cells lack the enzymes required to convert 5-fluorocytosine to 5 -fluorouracil; therefore, the drug is less toxic than 5-fluorouracil to patients. The potential of 5-fluorocytosine as an antibacterial was identified from a drug repurposing screen. 5-Fluorocytosine was identified as a compound that inhibited production of a number of virulence factors of $P$. aeruginosa (Imperi et al., 2013, 2019). 5-Fluorocytosine suppressed $P$. aeruginosa pathogenicity in a mouse model of pulmonary infection (Imperi et al., 2013) as well as in a Caenorhabditis elegans infection model (Kirienko et al., 2016). 5-Fluorocytosine needs to be deaminated to 5 -fluorouracil to inhibit virulence factor production of $P$. aeruginosa (Imperi et al., 2013, 2019).

\section{Idoxuridine}

Idoxuridine (5-iodo- 2 '-deoxyuridine; Figure 2B) was the first antiviral drug introduced to the clinic (Kaufman, 1962); nowadays, it is mostly used in the therapy of herpetic simplex keratitis (Wilhelmus, 2010). At high concentrations, idoxuridine is cytotoxic because it competitively inhibits various enzymes, including $\mathrm{TK}$, and when triphosphorylated can become incorporated into DNA (Figure 1; Prusoff et al., 1979). The exact effect of the incorporation of triphosphorylated idoxuridine into DNA is unknown.

Idoxuridine alone had no antimicrobial properties against a range of clinically important pathogens tested (Zander et al., 2010a). However, when used in conjunction with SXT, a combination of trimethoprim and sulfamethoxazole that inhibits tetrahydrofolic acid synthesis, idoxuridine significantly enhanced the antimicrobial properties of SXT against most of the bacterial species tested, including K. pneumoniae, S. aureus, and S. pyogenes (Zander et al., 2010a,b). Tetrahydrofolic acid is a critical bacterial cofactor of thymidine synthesis, and thus, DNA synthesis (Sköld, 2009) and inhibition of TK by idoxuridine evidently enhanced the effects of SXT in inhibiting DNA synthesis. This was the case even in the presence of extracellular thymidine, which would be present during infections and ameliorate the effects of SXT (Zander et al., 2010a,b).

While the combination of idoxuridine and SXT has promise for clinical application, extensive clinical studies will need to be performed. The use of idoxuridine is severely limited because of its cytotoxicity, and its use is largely limited to topical applications, where toxicity is not significant (Boston Interhospital Virus Study Group and NIAID-Sponsored Cooperative Antiviral Clinical Study, 1975; Yolton and Haesart, 2008). 
Although idoxuridine was effective against $S$. aureus in vitro at concentrations below those known to be cytotoxic (Zander et al., 2010a), it is unclear whether such low concentrations would improve the bactericidal effect of drugs like SXT in vivo.

\section{PURINE ANALOGUES}

\section{Thiopurines}

In contrast to pyrimidines, for which halogenated analogues have been characterized, purine analogues with potential application as antimicrobials are predominantly thio-derivatives. Currently, the predominant clinical uses for thiopurines are as anticancer, immunosuppressant, and anti-inflammatory agents. In particular, they are important therapeutic agents for acute leukemias and for inflammatory bowel disease (Galmarini et al., 2002; Cooper and Brown, 2015; Hanauer et al., 2019). Compounds from the thiopurine family (Figure 2D) have antibacterial activity. As mercaptopurine has in vitro activity against Mycobacterium avium subspecies paratuberculosis and Corynebacterium species (Greenstein et al., 2007; Shin and Collins, 2008; Liu et al., 2017), 6-thioguanosine 5'-triphosphate (thio-dGTP) can inhibit spore germination of Bacillus anthracis in an infected macrophage cell line (Akoachere et al., 2007; Alvarez et al., 2010), and thioguanine can inhibit in vitro growth of Mycoplasma pneumoniae (Sun and Wang, 2013).

Mercaptopurine is an analogue of hypoxanthine, and thioguanine is an analogue of guanine. Mercaptopurine and thioguanine require phosphorylation before they can exert their therapeutic effects. Both mercaptopurine and thioguanosine are metabolized intracellularly to the active thio-dGTP (Lennard, 1992), although mercaptopurine is also metabolized to other nucleoside derivatives. Thio-dGTP exerts its cytotoxic effect on cells primarily by becoming incorporated into DNA (Figure 1), resulting in local changes to the DNA structure, largely because it forms an unstable base pair with deoxycytidine (Somerville et al., 2003; de Boer et al., 2007; Karran and Attard, 2008). This change in structure can lead to DNA breaks and also inhibition of DNA replication (Somerville et al., 2003). Thiopurine derivatives can also inhibit enzymes involved in de novo purine synthesis (Figure 1; Galmarini et al., 2002). In eukaryotic cells, thio-dGTP is also known to interfere with secondary messengers and energy carrying processes, competing with natural guanosine triphosphate (de Boer et al., 2007).

M. avium ssp. paratuberculosis may be an environmental trigger for Crohn's disease or even a causative agent of the disease (Greenstein and Collins, 2004). Mercaptopurine is commonly used to induce and maintain remission of Crohn's disease. The effect of mercaptopurine on the growth of M. avium ssp. paratuberculosis was therefore tested, and the drug was found to inhibit growth in vitro (Greenstein et al., 2007; Shin and Collins, 2008), although in vivo experiments have yet to be carried out.

Inosine is an important germinant of B. anthracis in vitro. Inosine analogues and guanosine analogues were tested for their ability to block in vitro germination of spores
(Akoachere et al., 2007). Thio-dGTP was the only compound identified as being effective at preventing spore germination. The ability of thio-dGTP to prevent necrosis in murine macrophages infected with $B$. anthracis was subsequently tested (Alvarez et al., 2010). Thio-dGTP and its parent compound 6-thioguanine were the most effective analogues and were able to protect cells from necrosis even after the time point at which $100 \%$ cell death normally occurs (Alvarez et al., 2010). While the activity of thio-dGTP as an antigerminant is promising, spore germination in vivo is a complex process dependent upon numerous factors, and further studies of the efficacy of thio-dGTP are required.

\section{METABOLISM AND MECHANISMS OF ACTION OF NUCLEOSIDE ANALOGUES IN BACTERIA}

Pyrimidine and purine analogues utilize the same pathways as their natural counterparts and therefore compete for both uptake and metabolism (Sun and Wang, 2013). Typically, nucleobase and nucleoside analogues enter bacterial cells through membrane transporters and then are cycled through the nucleotide salvage pathway, where they are activated by deoxyribonucleoside kinases (dNKs). dNKs perform the first committed reaction in the salvage pathway, namely the phosphorylation of deoxyribonucleosides (Figure 1). In bacteria, the metabolism and mechanism of action of pyrimidine and purine analogues have significant differences and will be further discussed separately.

\section{Pyrimidine Analogues}

Early work on the metabolism and mechanism of action of pyrimidine analogues in bacteria demonstrated the importance of the enzymes that metabolize them, such as dNKs. Thymidine kinase (TK), a dNK, was shown to be necessary for activation of zidovudine in bacteria and subsequent incorporation of activated zidovudine into the DNA (Elwell et al., 1987). Since the identification of $\mathrm{TK}$ as being important for the activity of zidovudine, the involvement of dNKs and other enzymes on the metabolism and activity of pyrimidine analogues has been demonstrated.

dNK genes are differentially distributed among different bacterial species (Saito and Tomioka, 1984; Sandrini et al., 2007b; Konrad et al., 2012), and this differential distribution explains in part species-specific susceptibilities to nucleoside analogues. For example, $P$. aeruginosa lacks TK activity and is not susceptible to many pyrimidine analogues that have activity against other Gram-negative bacteria. Additionally, the presence of different dNKs in different species explains differences in responses to gemcitabine, which is found to be effective predominantly against Gram-positive bacteria. E. coli that lacks deoxyadenosine kinase is not usually susceptible to gemcitabine but is sensitive to this compound when expressing deoxyadenosine kinases from Gram-positive bacteria such as S. aureus, S. pyogenes, and B. cereus (Sandrini et al., 2007a,b). 
Zidovudine is effective against some Gram-negative but not Gram-positive species (Sandrini et al., 2007a). The basis for this was explored using an E. coli mutant lacking TK. This mutant was resistant to zidovudine but became sensitized when transformed with TK genes from either Gram-negative species or zidovudine-resistant Gram-positive species such as Bacillus cereus and Listeria monocytogenes (Elwell et al., 1987; Sandrini et al., 2007a,b). These findings suggest that resistance in the Gram-positive species is not a result of lack of TK activity toward zidovudine, but instead that the monophosphate form of zidovudine resulting from the action of TK is a poor substrate for subsequent enzymes such as thymidylate kinase (Sandrini et al., 2007a). TKs are also important for the activity of other fluorinated pyrimidines such as floxuridine in U. urealyticum (Carnrot et al., 2003). Floxuridine and trifluridine are substrates for $U$. urealyticum $\mathrm{TK}$ and are converted to the monophosphate forms that may be further metabolized and incorporated into DNA (Sun and Wang, 2013). The monophosphate forms of floxuridine and trifluridine may also inhibit thymidylate synthase.

An important part of the mechanism of action of nucleoside analogues in cancers and viruses is the incorporation of their metabolites into nucleic acids. There is evidence that this incorporation is important in bacteria as well. Metabolites of 5-fluorouracil become incorporated into E. coli RNA and to a lesser extent DNA (Warner and Rockstroh, 1980). The proportion of fluorinated uracil derivatives in the DNA increased significantly when the enzymes responsible for the removal of deoxyuridine triphosphate (UTP) from DNA were absent, indicating that this mechanism has a role in the removal of fluorinated UTP from DNA (Warner and Rockstroh, 1980). Fluorinated metabolites were also detected in the nucleic acids of Mycobacterium tuberculosis (Singh et al., 2015). In M. tuberculosis, treatment with 5-fluorouracil also led to an upregulation of DNA damage response genes proposed to be a result of DNA breakages caused by incorporation of fluorinated UTP (Singh et al., 2015).

An effect of fluorinated pyrimidines independent of nucleic acid incorporation has also been observed. Treatment with 5-fluorouracil caused E. coli to become osmotically sensitive, leading to a rapid loss of bacterial viability (Tomasz and Borek, 1959), an effect proposed to be due to metabolites of 5-fluorouracil affecting UDP-linked cell-wall precursors (Tomasz and Borek, 1960, 1962). 5-Fluorouracil partially inhibited peptidoglycan biosynthesis in S. aureus, leading to accumulation of fluorinated cell-wall precursors (Rogers and Perkins, 1960; Stickgold and Neuhaus, 1967). Accumulation of fluorinated cell-wall precursors also occurred in 5-fluorouracil-treated Mycobacterium tuberculosis (Singh et al., 2015).

\section{Purine Analogues}

One of the first enzymes in the purine salvage pathway is hypoxanthine guanine phosphoribosyl transferase (HPRT) (Figure 1). Thioguanosine strongly inhibited the growth of M. pneumoniae, and HPRT is a target of this analogue (Sun and Wang, 2013). Thioguanosine strongly inhibited uptake of hypoxanthine and guanosine and their subsequent incorporation into DNA and RNA. Thioguanosine also strongly inhibited activity of M. pneumoniae HPRT with either of its natural substrates, hypoxanthine and guanosine (Figure 1). Thioguanosine inhibited M. pneumoniae HPRT much more effectively than human HPRT. This likely reflects structural differences between humans and M. pneumoniae HPRT leading to differences in binding of thioguanosine; these differences may allow for designing pathogen-specific HPRT inhibitors (Sun and Wang, 2013).

\section{LIMITATIONS OF NUCLEOSIDE ANALOGUES AS ANTIBIOTICS}

\section{Toxicity}

While the safety profiles of clinically used nucleoside analogues are generally favorable, some of these drugs do have side effects. Nucleoside analogues, especially those used as anticancer agents, are often not specific for their target cells and also affect healthy human cells. Therefore, the use of this class of drugs is associated with a number of side effects. Many side effects are mild and do not require cessation of treatment, but some side effects are severe. For example, some analogues such as trifluridine and idoxuridine are only used topically as they have severe side effects when used systemically. While severe side effects as a result of treatment with nucleoside analogues are generally rare, they will need to be considered if nucleoside analogues are to have clinical application as antibiotics.

One of the most common significant side effects of nucleoside analogues is myelosuppression. Myelosuppression is a decrease in the production of blood cells in bone marrow and can affect all types of blood cells. A serious type of myelosuppression caused by chemotherapy is neutropenia, which is the reduction of leukocytes. Neutropenia predisposes patients to infections and is the dose-limiting factor in many therapeutic regimens with nucleoside analogues, including gemcitabine, 5-fluorouracil, and floxuridine, and the thiopurines (Galmarini et al., 2002). Myelosuppression is also a dose-limiting toxicity in the use of 5-fluorocytosine as an antifungal agent. Even though 5-fluorocytosine is a prodrug and human cells lack the enzyme needed to convert it to 5 -fluorouracil, when serum concentration of 5-fluorocytosine reaches $100 \mu \mathrm{g} / \mathrm{ml}$ or higher, toxicities similar to those seen in patients taking 5-fluorouracil can occur (Kauffman and Frame, 1977; Harris et al., 1986). Why these toxicities occur is unclear, although there is evidence that the host gut microbiota may convert 5-fluorocytosine to 5-fluorouracil, which can cause myelosuppression (Harris et al., 1986; Vermes et al., 2003). Of the drugs discussed in this review, idoxuridine highlights the problem myelosuppression may cause when nucleoside analogues are used as antibiotics. While idoxuridine is used to treat herpetic simplex keratitis of the eye, it has been found to worsen and slow healing of bacterial infections of the eye (Yamaguchi et al., 1979). Thus, nucleoside analogues that need to be administered at doses that cause myelosuppression may be contraindicated for use against bacterial infections.

Another side effect is pulmonary toxicity, commonly associated with gemcitabine use. Gemcitabine causes a range of pulmonary 
toxicities, from those that are mild and do not require stopping treatment, to rare but severe side effects, like pulmonary fibrosis and respiratory distress syndrome (Chi et al., 2012). It is unclear why gemcitabine has this effect, but it has been proposed that pulmonary toxicity occurs more frequently in patients who have underlying pulmonary disease or in patients who are concomitantly taking therapeutics that can also cause pulmonary toxicity (Gupta et al., 2002). The concentrations of gemcitabine that inhibited the growth of $S$. aureus in vitro were lower than the plasma concentrations of gemcitabine in cancer patients, suggesting that lower doses than are used in oncology may be effective in treating infections, potentially reducing side effects of gemcitabine (Jordheim et al., 2012).

While it is proposed that nucleoside analogues would be effective against bacteria at concentrations that make them unlikely to cause myelosuppression or other serious side effects (Walz et al., 2010; Zander et al., 2010a; Jordheim et al., 2012), toxicity remains a serious consideration that must be resolved before these drugs can be used as antibacterials. Treatment of bacterial infections with compounds, which at high serum concentrations can have immunomodulatory or toxic effects, could potentially result in the drug exacerbating the infection it was intended to treat. The examples described above highlight the fact that a significant amount of investigation into the safety of nucleoside analogues as antibiotics will have to be performed to ensure bacterial infections are not worsened by administration of analogues.

A potential way to overcome any toxic effects is to search for nucleoside analogues that are more specific for their bacterial targets. Characterization of the substrate specificities of different bacterial dNKs, which are responsible for the activation of many nucleoside analogues in bacteria, suggests that it may be possible to design analogues specific for bacterial dNKs (Sandrini et al., 2007a; Doleans-Jordheim et al., 2011).

\section{Resistance}

As found for classical antibiotics, resistance can arise when using nucleoside analogues as antibiotics. Resistance to zidovudine and gemcitabine has been studied in detail. After short-term exposure to zidovudine, Salmonella and E. coli strains have been found develop stable high-level resistance both in vitro and in vivo (Lewin et al., 1990a,b; Doleans-Jordheim et al., 2011). Zidovudine-resistant E. coli has been isolated from HIV/ AIDS patients taking zidovudine as antiretroviral therapy, whereas E. coli isolated from control samples was susceptible (Lewin et al., 1990b, 1991).

A mechanism for resistance to zidovudine was first proposed by Lewin et al. (1990a). It was found that zidovudine-resistant strains of E. coli and S. typhimurium were unable to incorporate radiolabeled thymidine into their chromosomal DNA, and that this incorporation was still prevented in the presence of a membrane permeabilizer. This suggested that in these bacteria, resistance was due not to inhibition of uptake but rather to loss of TK activity (Lewin et al., 1990a). As TKs have been shown to be important for conversion of nucleoside analogues into their active forms, this may provide an explanation for the observed resistance. Zidovudine has been found to induce mutations in E. coli (Doleans-Jordheim et al., 2011). Because of the apparent importance of TK on the metabolism of zidovudine (Sandrini et al., 2007a,b), Doleans-Jordheim et al. (2011) compared the sequence of the TK-encoding gene from resistant strains and susceptible strains. All 10 strains analyzed had changes to the sequences of the gene that would result in changes to the TK protein, including amino acid changes and premature stop codons.

In addition to zidovudine resistance via a non-functional TK enzyme, there appears to be another mechanism of resistance. Some resistant bacteria, like staphylococci, do have functional TK enzymes (Lewin et al., 1990a). The reason why these bacterial strains are zidovudine resistant is not clear, but it is possible that in such strains, zidovudine does not act as a substrate for TK or DNA polymerase and is thus not incorporated into nucleic acids (Lewin et al., 1990a).

Resistance to gemcitabine has also been observed. Jordheim et al. (2012) observed regrowth of S. aureus treated with gemcitabine, even at concentrations significantly above the determined minimum inhibitory concentration values. Mutational frequencies were found to be similar to those found for classical antibiotics. Most of the resistant mutants had mutations in the gene encoding deoxyadenosine kinase that is thought to activate gemcitabine (Jordheim et al., 2012). It was concluded that mutations of deoxyadenosine kinase were important for conferring resistance to gemcitabine, although as some resistant mutants had no mutations in the dNK genes, there are likely to be other mechanisms involved. The induced resistance was found to be highly stable (Jordheim et al., 2012).

While resistance to both zidovudine and gemcitabine arises readily, this could be prevented at least in part either by coadministering with other drugs or possibly by modifying the drug itself. Zidovudine has been shown to have synergistic activity with gentamicin and amikacin (Doleans-Jordheim et al., 2011). Gemcitabine has synergy with gentamicin, and combinations of drugs may help slow the emergence of resistance (Jordheim et al., 2012).

\section{CONCLUSIONS AND FUTURE PERSPECTIVES}

Nucleoside analogues are widely used as effective therapeutics for a variety of diseases and thus make an interesting focus on repurposing studies. Studies to date have yielded promising data on the efficacy of analogues as antibacterial agents, highlighting their potential for use in treating bacterial infections. This potential will become increasingly important as the numbers of multidrug-resistant bacteria continue to rise. Nonetheless, to the best of our knowledge, no nucleoside analogues are currently approved for treating bacterial infections, and so they are not used even in cases where no other options are available.

What are the gaps in current knowledge that are barriers to the use of nucleoside analogues in treating bacterial infections? First and foremost, our review highlights the paucity of studies investigating the effectiveness of nucleoside analogues in treating infections in animal models, information that is needed to 
determine doses that would be needed to treat infections. This information is particularly crucial for nucleoside analogues that commonly have adverse side effects when used at higher doses, such as in cancer therapy. Second, although the nucleoside analogues discussed here are approved for use in people, clinical trials will be necessary to test their effectiveness in treating bacterial infections and costs are associated with these. Third, the antibacterial modes of action of many nucleoside analogues are not fully understood. Overcoming this knowledge gap may facilitate strategies for better targeting nucleoside analogues as inhibitors of infection while minimizing toxicity to patients. Lastly, although investigations of resistance to nucleoside analogues have been carried out in a few cases, a comprehensive picture is lacking and it is not known how readily resistance might arise during infection. Examples described here of the use of nucleoside analogues in combination with other antibiotics, or as inhibitors of virulence rather than of bacterial growth, suggest strategies that may minimize the development of resistance.

The increasing emergence of multidrug-resistant bacteria capable of causing severe, life-threatening infections provides a hugely powerful imperative to develop new approaches for

\section{REFERENCES}

Akoachere, M., Squires, R. C., Nour, A. M., Angelov, L., Brojatsch, J., and AbelSantos, E. (2007). Identification of an in vivo inhibitor of Bacillus anthracis spore germination. J. Biol. Chem. 282, 12112-12118. doi: 10.1074/jbc.M611432200

Alvarez, Z., Lee, K., and Abel-Santos, E. (2010). Testing nucleoside analogues as inhibitors of Bacillus anthracis spore germination in vitro and in macrophage cell culture. Antimicrob. Agents Chemother. 54, 5329-5336. doi: 10.1128/ AAC.01029-10

Alvarez, P., Marchal, J. A., Boulaiz, H., Carrillo, E., Velez, C., Rodriguez-Serrano, F., et al. (2012). 5-Fluorouracil derivatives: a patent review. Expert Opin. Ther Pat. 22, 107-123. doi: 10.1517/13543776.2012.661413

Bennett, J. E. (1977). Flucytosine. Ann. Intern. Med. 86, 319-322. doi: 10.7326/0003-4819-86-3-319

Boston Interhospital Virus Study Group and NIAID-Sponsored Cooperative Antiviral Clinical Study (1975). Failure of high dose 5-iodo-2'-deoxyuridine in the therapy of herpes simplex virus encephalitis. Evidence of unacceptable toxicity. N. Engl. J. Med. 292, 599-603. doi: 10.1056/NEJM197503202921201

Brown, D. (2015). Antibiotic resistance breakers: can repurposed drugs fill the antibiotic discovery void? Nat. Rev. Drug Discov. 14, 821-832. doi: 10.1038/ $\operatorname{nrd} 4675$

Burris, H. A., Moore, M. J., Andersen, J., Green, M. R., Rothenberg, M. L., Madiano, M. R., et al. (1997). Improvements in survival and clinical benefit with gemcitabine as first-line therapy for patients with advanced pancreas cancer: a randomized trial. J. Clin. Oncol. 15, 2403-2413. doi: 10.1200/ JCO.1997.15.6.2403

Carnrot, C., Wehelie, R., Eriksson, S., Bolske, G., and Wang, L. Y. (2003). Molecular characterization of thymidine kinase from Ureaplasma urealyticum: nucleoside analogues as potent inhibitors of mycoplasma growth. Mol. Microbiol. 50, 771-780. doi: 10.1046/j.1365-2958.2003.03717.x

Carter, G. T., and McDonald, L. (2014). "Uridyl peptide antibiotics: developments in biosynthesis and medicinal chemistry" in Antimicrobials. eds. F. Marinelli, and O. Genilloud (Berlin: Springer-Verlag), 177-191. doi: 10.1007/978-3-662-45786-3_9

Casado, J. L., Valdezate, S., Calderon, C., Navas, E., Frutos, B., Guerrero, A., et al. (1999). Zidovudine therapy protects against Salmonella bacteremia recurrence in human immunodeficiency virus-infected patients. J. Infect. Dis. 179, 1553-1556. doi: 10.1086/314749

Chi, D. C., Brogan, F., Turenne, I., Zelonis, S., Schwartz, L., and Saif, M. W. (2012). Gemcitabine-induced Pulmonary Toxicity. Anticancer Res. 32, 4147-4149. treating bacterial infections. The research reviewed here highlights the potential for use of nucleoside analogues in situations where classical antibiotics fail. With the current requirement for more antibiotics, it will be very important to fill the knowledge gaps described above, so that nucleoside antibiotics can provide a clinically usable tool in the treatment of bacterial infections.

\section{AUTHOR CONTRIBUTIONS}

JT and IL reviewed the literature. JT prepared the figures. JT and IL wrote the manuscript.

\section{FUNDING}

JT was the recipient of a $\mathrm{PhD}$ scholarship from the University of Otago. Research in the authors' laboratory is supported by the Otago Medical Research Foundation (grant AG330), the New Zealand Health Research Council (17/372), Cure Kids New Zealand (3574), and Cystic Fibrosis New Zealand.

Cooper, S. L., and Brown, P. A. (2015). Treatment of pediatric acute lymphoblastic leukemia. Pediatr. Clin. N. Am. 62, 61-73. doi: 10.1016/j.pcl.2014.09.006

Cooper, D. L., and Lovett, S. T. (2011). Toxicity and tolerance mechanisms for azidothymidine, a replication gap-promoting agent, in Escherichia coli. DNA Repair 10, 260-270. doi: 10.1016/j.dnarep.2010.11.007

de Boer, N. K. H., Van Bodegraven, A. A., Jharap, B., de Graaf, P., and Mulder, C. J. J. (2007). Drug insight: pharmacology and toxicity of thiopurine therapy in patients with IBD. Nat. Clin. Pract. Gastroenterol. Hepatol. 4, 686-694. doi: $10.1038 /$ ncpgasthep 1000

Doleans-Jordheim, A., Bergeron, E., Bereyziat, F., Ben-Larbi, S., Dumitrescu, O., Mazoyer, M. A., et al. (2011). Zidovudine (AZT) has a bactericidal effect on enterobacteria and induces genetic modifications in resistant strains. Eur. J. Clin. Microbiol. Infect. Dis. 30, 1249-1256. doi: 10.1007/ s10096-011-1220-3

Elwell, L. P., Ferone, R., Freeman, G. A., Fyfe, J. A., Hill, J. A., Ray, P. H., et al. (1987). Antibacterial activity and mechanism of action of 3'-azido-3'-deoxythymidine (Bw-A509u). Antimicrob. Agents Chemother. 31, 274-280. doi: 10.1128/AAC.31.2.274

Falagas, M. E., Voulgaris, G. L., Tryfinopoulou, K., Giakkoupi, P., Kyriakidou, M., Vatopoulos, A., et al. (2019). Synergistic activity of colistin with azidothymidine against colistin-resistant Klebsiella pneumoniae clinical isolates collected from inpatients in Greek hospitals. Int. J. Antimicrob. Agents. doi: 10.1016/j. ijantimicag.2019.02.021

Francis, P., and Walsh, T. J. (1992). Evolving role of flucytosine in immunocompromised patients - new insights into safety, pharmacokinetics, and antifungal therapy. Clin. Infect. Dis. 15, 1003-1018. doi: 10.1093/clind/15.6.1003

Furman, P. A., Fyfe, J. A., Stclair, M. H., Weinhold, K., Rideout, J. L., Freeman, G. A., et al. (1986). Phosphorylation of 3'-azido-3'-deoxythymidine and selective interaction of the $5^{\prime}$-triphosphate with human-immunodeficiencyvirus reverse-transcriptase. Proc. Natl. Acad. Sci. USA 83, 8333-8337. doi: 10.1073/pnas.83.21.8333

Galmarini, C. M., Mackey, J. R., and Dumontet, C. (2002). Nucleoside analogues and nucleobases in cancer treatment. Lancet Oncol. 3, 415-424. doi: 10.1016/ S1470-2045(02)00788-X

Gavrish, E., Sit, C. S., Cao, S. G., Kandror, O., Spoering, A., Peoples, A., et al. (2014). Lassomycin, a ribosomally synthesized cyclic peptide, kills Mycobacterium tuberculosis by targeting the ATP-dependent protease ClpC1P1P2. Chem. Biol. 21, 509-518. doi: 10.1016/j.chembiol.2014.01.014

Ghannoum, M. A., and Rice, L. B. (1999). Antifungal agents: mode of action, mechanisms of resistance, and correlation of these mechanisms with bacterial resistance. Clin. Microbiol. Rev. 12, 501-517. doi: 10.1128/CMR.12.4.501 
Greenstein, R. J., and Collins, M. T. (2004). Emerging pathogens: is Mycobacterium avium subspecies paratuberculosis zoonotic? Lancet 364, 396-397. doi: 10.1016/ S0140-6736(04)16781-0

Greenstein, R. J., Su, L., Haroutunian, V., Shahidi, A., and Brown, S. T. (2007). On the action of methotrexate and 6-mercaptopurine on $M$. avium subspecies paratuberculosis. PLoS One 2:e161. doi: 10.1371/journal.pone.0000516

Gupta, N., Ahmed, I., Steinberg, H., Patel, D., Nissel-Horowitz, S., and Mehrotra, B. (2002). Gemcitabine-induced pulmonary toxicity-case report and review of the literature. Am. J. Clin. Oncol. 25, 96-100. doi: 10.1097/00000421-200202000-00021

Hanauer, S. B., Sandborn, W. J., and Lichtenstein, G. R. (2019). Evolving considerations for thiopurine therapy for inflammatory bowel diseases-a clinical practice update: commentary. Gastroenterology 156, 36-42. doi: 10.1053/j.gastro.2018.08.043

Harris, B., Manning, B., Federle, T., and Diasio, R. (1986). Conversion of 5-fluorocytosine to 5-fluorouracil by human intestinal microflora. Fed. Proc. 45:443. doi: 10.1128/AAC.29.1.44

Heidelberger, C., Chaudhuri, N. K., Danneberg, P., Mooren, D., Griesbach, L., Duschinsky, R., et al. (1957). Fluorinated pyrimidines, a new class of tumourinhibitory compounds. Nature 179, 663-666. doi: 10.1038/179663a0

Heinemann, V., Hertel, L. W., Grindey, G. B., and Plunkett, W. (1988). Comparison of the cellular pharmacokinetics and toxicity of $2^{\prime}, 2^{\prime}$-difluorodeoxycytidine and 1-beta-D-arabinofuranosylcytosine. Cancer Res. 48, 4024-4031.

Herrmann, J. L., and Lagrange, P. H. (1992). Intracellular activity of zidovudine (3'-azido-3'-deoxythymidine, Azt) against Salmonella typhimurium in the macrophage cell line-J774-2. Antimicrob. Agents Chemother. 36, 1081-1085. doi: 10.1128/AAC.36.5.1081

$\mathrm{Hu}$, Y., Liu, Y., and Coates, A. (2019). Azidothymidine produces synergistic activity in combination with colistin against antibiotic-resistant Enterobacteriaceae. Antimicrob. Agents Chemother. 63:e01630-18. doi: 10.1128/ AAC.01630-18

Huang, P., and Plunkett, W. (1995). Induction of apoptosis by gemcitabine. Semin. Oncol. 22, 19-25.

Imperi, F., Fiscarelli, E. V., Visaggio, D., Leoni, L., and Visca, P. (2019). Activity and impact on resistance development of two antivirulence fluoropyrimidine drugs of Pseudomonas aeruginosa. Front. Cell. Infect. Microbiol. 9:49. doi: 10.3389/fcimb.2019.00049

Imperi, F., Massai, F., Facchini, M., Frangipani, E., Visaggio, D., Leoni, L., et al. (2013). Repurposing the antimycotic drug flucytosine for suppression of Pseudomonas aeruginosa pathogenicity. Proc. Natl. Acad. Sci. USA 110:16693. doi: $10.1073 /$ pnas. 1222706110

Ishii, H., Furuse, J., Nagase, M., and Yoshino, M. (2005). Impact of gemcitabine on the treatment of metastatic pancreatic cancer. J. Gastroenterol. Hepatol. 20, 62-66. doi: 10.1111/j.1440-1746.2004.03487.x

Jordheim, L. P., Ben Larbi, S., Fendrich, O., Ducrot, C., Bergeron, E., Dumontet, C., et al. (2012). Gemcitabine is active against clinical multiresistant Staphylococcus aureus strains and is synergistic with gentamicin. Int. J. Antimicrob. Agents 39, 444-447. doi: 10.1016/j.ijantimicag.2012.01.019

Jordheim, L. P., Durantel, D., Zoulim, F., and Dumontet, C. (2013). Advances in the development of nucleoside and nucleotide analogues for cancer and viral diseases. Nat. Rev. Drug Discov. 12, 447-464. doi: 10.1038/ $\operatorname{nrd} 4010$

Karran, P., and Attard, N. (2008). Thiopurines in current medical practice: molecular mechanisms and contributions to therapy-related cancer. Nat. Rev. Cancer 8, 24-36. doi: 10.1038/nrc2292

Kauffman, C. A., and Frame, P. T. (1977). Bone-marrow toxicity associated with 5-fluorocytosine therapy. Antimicrob. Agents Chemother. 11, 244-247. doi: 10.1128/AAC.11.2.244

Kaufman, H. E. (1962). Clinical cure of herpes simplex keratitis by 5-iodo-2deoxyuridine. Proc. Soc. Exp. Biol. Med. 109, 251-252. doi: 10.3181/00379727-109-27169

Keith, B. R., White, G., and Wilson, H. R. (1989). Invivo efficacy of zidovudine (3'-azido-3'-deoxythymidine) in experimental gram-negative-bacterial infections. Antimicrob. Agents Chemother. 33, 479-483. doi: 10.1128/ AAC.33.4.479

Kirienko, D. R., Revtovich, A. V., and Kirienko, N. V. (2016). A high-content, phenotypic screen identifies fluorouridine as an inhibitor of pyoverdine biosynthesis and Pseudomonas aeruginosa virulence. mSphere 1:e00217-16. doi: $10.1128 / \mathrm{mSphere} .00217-16$
Konrad, A., Yarunova, E., Tinta, T., Piskur, J., and Liberles, D. A. (2012). The global distribution and evolution of deoxyribonucleoside kinases in bacteria. Gene 492, 117-120. doi: 10.1016/j.gene.2011.10.039

Koszytkowska-Stawinska, M., and Buchowicz, W. (2014). Multicomponent reactions in nucleoside chemistry. Beilstein J. Org. Chem. 10, 1706-1732. doi: $10.3762 /$ bjoc. 10.179

Lennard, L. (1992). The clinical-pharmacology of 6-mercaptopurine. Eur. J. Clin. Pharmacol. 43, 329-339. doi: 10.1007/BF02220605

Lewin, C. S., Allen, R. A., and Amyes, S. G. B. (1990a). Mechanisms of Zidovudine Resistance in Bacteria. J. Med. Microbiol. 33, 235-238. doi: 10.1099/00222615-33-4-235

Lewin, C. S., Watt, B., and Naden, M. (1991). Zidovudine resistance in clinically significant bacterial isolates from aids patients. J. Antimicrob. Chemother 28, 778-780. doi: 10.1093/jac/28.5.778

Lewin, C. S., Watt, B., Paton, R., and Amyes, S. G. B. (1990b). Isolation of zidovudine resistant Escherichia coli from AIDS patients. FEMS Microbiol. Lett. 70, 141-144. doi: 10.1111/j.1574-6968.1990.tb13967.x

Lewis, K. (2013). Platforms for antibiotic discovery. Nat. Rev. Drug Discov. 12, 371-387. doi: 10.1038/nrd3975

Lewis, W., Gonzalez, B., Chomyn, A., and Papoian, T. (1992). Zidovudine induces molecular, biochemical, and ultrastructural-changes in rat skeletalmuscle mitochondria. J. Clin. Investig. 89, 1354-1360. doi: 10.1172/JCI115722

Lewis, W., Grupp, I. L., Grupp, G., Hoit, B., Morris, R., Samarel, A. M., et al. (2000). Cardiac dysfunction occurs in the HIV-1 transgenic mouse treated with zidovudine. Lab. Investig. 80, 187-197. doi: 10.1038/labinvest.3780022

Ling, L. L., Schneider, T., Peoples, A. J., Spoering, A. L., Engels, I., Conlon, B. P., et al. (2015). A new antibiotic kills pathogens without detectable resistance. Nature 517, 455-459. doi: 10.1038/nature14098

Liu, F., Ma, R., Riordan, S. M., Grimm, M. C., Liu, L., Wang, Y., et al. (2017). Azathioprine, mercaptopurine, and 5-aminosalicylic acid effect the growth of IBD-associated campylobacter species and other enteric microbes. Front. Microbiol. 8:527. doi: 10.3389/fmicb.2017.00527

Livermore, D. M. (2011). Discovery research: the scientific challenge of finding new antibiotics. J. Antimicrob. Chemother. 66, 1941-1944. doi: 10.1093/jac/ dkr262

Mini, E., Nobili, S., Caciagli, B., Landini, I., and Mazzei, T. (2006). Cellular pharmacology of gemcitabine. Ann. Oncol. 17, V7-V12. doi: 10.1093/annonc/ mdj941

Miro-Canturri, A., Ayerbe-Algaba, R., and Smani, Y. (2019). Drug repurposing for the treatment of bacterial and fungal infections. Front. Microbiol. 10:41. doi: $10.3389 /$ fmicb.2019.00041

Moroni, G. N., Bogdanov, P. M., and Brinon, M. C. (2002). Synthesis and in vitro antibacterial activity of novel 5'-O-analog derivatives of zidovudine as potential prodrugs. Nucleosides Nucleotides Nucleic Acids 21, 231-241. doi: $10.1081 / \mathrm{NCN}-120003288$

Ng, S. M. S., Sioson, J. S. P., Yap, J. M., Ng, F. M., Ching, H. S. V., Teo, J. W. P., et al. (2018). Repurposing Zidovudine in combination with Tigecycline for treating carbapenem-resistant Enterobacteriaceae infections. Eur. J. Clin. Microbiol. Infect. Dis. 37, 141-148. doi: 10.1007/s10096-017-3114-5

Niu, G. Q., and Tan, H. R. (2015). Nucleoside antibiotics: biosynthesis, regulation, and biotechnology. Trends Microbiol. 23, 110-119. doi: 10.1016/j.tim.2014.10.007

O'Neill, J.C. (2014). Review on antimicrobial resistance. Antimicrobial resistance: tackling a crisis for the health and wealth of nations. (United Kingdom Government Report). (Accessed March 21, 2019).

Peyclit, L., Baron, S. A., Yousfi, H., and Rolain, J. M. (2018). Zidovudine: a salvage therapy for mcr-1 plasmid-mediated colistin-resistant bacterial infections? Int. J. Antimicrob. Agents 52, 11-13. doi: 10.1016/j.ijantimicag.2018.03.012

Plunkett, W., Huang, P., and Gandhi, V. (1995). Preclinical characteristics of gemcitabine. Anti-Cancer Drugs 6, 7-13. doi: 10.1097/00001813199512006-00002

Prusoff, W. H., Chen, M. S., Fischer, P. H., Lin, T. S., Shiau, G. T., Schinazi, R. F., et al. (1979). Antiviral iodinated pyrimidine deoxyribonucleosides: 5-iodo- $2^{\prime}$ deoxyuridine; 5-iodo-2' -deoxycytidine; 5-iodo-5' -amino-2' - $^{\prime}$-dideoxyuridine. Pharmacol. Ther. 7, 1-34. doi: 10.1016/0163-7258(79)90023-8

Rangel-Vega, A., Bernstein, L. R., Mandujano-Tinoco, E. A., Garcia-Contreras, S. J., and Garcia-Contreras, R. (2015). Drug repurposing as an alternative for the treatment of recalcitrant bacterial infections. Front. Microbiol. 6:282. doi: $10.3389 /$ fmicb. 2015.00282 
Rice, L. B. (2009). The clinical consequences of antimicrobial resistance. Curr. Opin. Microbiol. 12, 476-481. doi: 10.1016/j.mib.2009.08.001

Rogers, H. J., and Perkins, H. R. (1960). 5-Fluorouracil and mucopeptide biosynthesis by Staphylococcus aureus. Biochem. J. 77, 448-459. doi: 10.1042/ bj0770448

Rutman, R. J., Cantarow, A., and Paschkis, K. E. (1954). The catabolism of uracil in vivo and in vitro. J. Biol. Chem. 210, 321-329.

Saito, H., and Tomioka, H. (1984). Thymidine kinase of bacteria-activity of the enzyme in actinomycetes and related organisms. J. Gen. Microbiol. 130, 1863-1870. doi: 10.1099/00221287-130-7-1863

Sandrini, M. P. B., Clausen, A. R., On, S. L. W., Aarestrup, F. M., MunchPetersen, B., and Piskur, J. (2007a). Nucleoside analogues are activated by bacterial deoxyribonucleoside kinases in a species-specific manner. J. Antimicrob. Chemother. 60, 510-520. doi: 10.1093/jac/dkm240

Sandrini, M. P. B., Shannon, O., Clausen, A. R., Bjorck, L., and Piskur, J. (2007b). Deoxyribonucleoside kinases activate nucleoside antibiotics in severely pathogenic bacteria. Antimicrob. Agents Chemother. 51, 2726-2732. doi: 10.1128/AAC.00081-07

Shin, S. J., and Collins, M. T. (2008). Thiopurine drugs azathioprine and 6-mercaptopurine inhibit Mycobacterium paratuberculosis growth in vitro. Antimicrob. Agents Chemother. 52:1208. doi: 10.1128/AAC.00125-08

Singh, V., Brecik, M., Mukherjee, R., Evans, J. C., Svetlikova, Z., Blasko, J., et al. (2015). The complex mechanism of antimycobacterial action of 5-fluorouracil. Chem. Biol. 22, 63-75. doi: 10.1016/j.chembiol.2014.11.006

Sköld, O. (2009). "Sulfonamides and trimethoprim" in Antimicrobial drug resistance. ed. D. L. Mayers (New York City: Humana Press), 259-269. doi: 10.1007/978-1-59745-180-2_23

Somerville, L., Krynetski, E. Y., Krynetskaia, N. F., Beger, R. D., Zhang, W. X., Marhefka, C. A., et al. (2003). Structure and dynamics of thioguaninemodified duplex DNA. J. Biol. Chem. 278, 1005-1011. doi: 10.1074/jbc. M204243200

Stickgold, R. A., and Neuhaus, F. C. (1967). On the initial stage in peptidoglycan synthesis. Effect of 5-fluorouracil substitution on phospho-N-acetylmuramylpentapeptide translocase (uridine 5'-phosphate). J. Biol. Chem. 242, 1331-1337.

Sun, R., and Wang, L. Y. (2013). Inhibition of Mycoplasma pneumoniae growth by FDA-approved anticancer and antiviral nucleoside and nucleobase analogs. BMC Microbiol. 13:184. doi: 10.1186/1471-2180-13-184

Tomasz, A., and Borek, E. (1959). An early phase in the bactericidal action of 5-fluorouracil on E. coli $\mathrm{K}_{12}$. Osmotic imbalance. Proc. Natl. Acad. Sci. USA. 45, 929-932. doi: 10.1073/pnas.45.7.929

Tomasz, A., and Borek, E. (1960). The mechanism of bacterial fragility produced by 5 -fluorouracil: the accumulation of cell wall precursors. Proc. Natl. Acad. Sci. USA 46, 324-327. doi: 10.1073/pnas.46.3.324

Tomasz, A., and Borek, E. (1962). The mechanism of an osmotic instability induced in E. coli K-12 by 5-fluorouracil. Biochemistry 1, 543-552. doi: 10.1021/bi00910a001

Ueda, A., Attila, C., Whiteley, M., and Wood, T. K. (2009). Uracil influences quorum sensing and biofilm formation in Pseudomonas aeruginosa and fluorouracil is an antagonist. Microb. Biotechnol. 2, 62-74. doi: 10.1111/j.1751-7915.2008.00060.x

Vanhaperen, V. W. T. R., Veerman, G., Vermorken, J. B., and Peters, G. J. (1993). 2',2'-difluoro-deoxycytidine (gemcitabine) incorporation into RNA and DNA of tumor-cell lines. Biochem. Pharmacol. 46, 762-766. doi: 10.1016/0006-2952(93)90566-F
Vanlancker, E., Vanhoecke, B., Smet, R., Props, R., and Van De Wiele, T. (2016). 5-fluorouracil sensitivity varies among oral micro-organisms. J. Med. Microbiol. 65, 775-783. doi: 10.1099/jmm.0.000292

Vermes, A., Kuijper, E. J., Guchelaar, H. J., and Dankert, J. (2003). An in vitro study on the active conversion of flucytosine to fluorouracil by microorganisms in the human intestinal microflora. Chemotherapy 49, 17-23. doi: $10.1159 / 000069784$

Walz, J. M., Avelar, R. L., Longtine, K. J., Carter, K. L., Mermel, L. A., Heard, S. O., et al. (2010). Anti-infective external coating of central venous catheters: a randomized, noninferiority trial comparing 5-fluorouracil with chlorhexidine/silver sulfadiazine in preventing catheter colonization. Crit. Care Med. 38, 2095-2102. doi: 10.1097/CCM.0b013e3181f265ba

Wambaugh, M. A., Shakya, V. P. S., Lewis, A. J., Mulvey, M. A., and Brown, J. C. S. (2017). High-throughput identification and rational design of synergistic small-molecule pairs for combating and bypassing antibiotic resistance. PLoS Biol. 15:e2001644. doi: 10.1371/journal.pbio.2001644

Warner, H. R., and Rockstroh, P. A. (1980). Incorporation and excision of 5-fluorouracil from deoxyribonucleic acid in Escherichia coli. J. Bacteriol. 141, 680-686.

Wehelie, R., Eriksson, S., and Wang, L. Y. (2004). Effect of fluoropyrimidines on the growth of Ureaplasma urealyticum. Nucleosides Nucleotides Nucleic Acids 23, 1499-1502. doi: 10.1081/NCN-200027714

Wilhelmus, K. R. (2010). Antiviral treatment and other therapeutic interventions for herpes simplex virus epithelial keratitis. Cochrane Database Syst. Rev. 8:CD002898. doi: 10.1002/14651858.CD002898.pub4

Winn, M., Goss, R. J. M., Kimura, K., and Bugg, T. D. H. (2010). Antimicrobial nucleoside antibiotics targeting cell wall assembly: recent advances in structure-function studies and nucleoside biosynthesis. Nat. Prod. Rep. 27, 279-304. doi: 10.1039/B816215H

Yamaguchi, K., Okumoto, M., Stern, G., Friedlaender, M., and Smolin, G. (1979). Idoxuridine and bacterial corneal infection. Am J. Ophthalmol. 87, 202-205. doi: 10.1016/0002-9394(79)90143-0

Yolton, D. P., and Haesart, S. P. (2008). "Anti-infective drugs" in Clinical ocular pharmacology. 2nd Edn, ed. J. D. Bartlett. (St Louis, MO: Butterworth-Heinemann), 175-220. doi: 10.1016/B978-0-7506-7576-5.50016-1

Zander, J., Besier, S., Ackermann, H., and Wichelhaus, T. A. (2010a). Synergistic antimicrobial activities of folic acid antagonists and nucleoside analogs. Antimicrob. Agents Chemother. 54, 1226-1231. doi: 10.1128/AAC.00705-09

Zander, J., Besier, S., Faetke, S., Saum, S. H., Muller, V., and Wichelhaus, T. A. (2010b). Antimicrobial activities of trimethoprim/sulfamethoxazole, 5-iodo-2' deoxyuridine and rifampicin against Staphylococcus aureus. Int. J. Antimicrob. Agents 36, 562-565. doi: 10.1016/j.ijantimicag.2010.08.007

Conflict of Interest Statement: The authors declare that the research was conducted in the absence of any commercial or financial relationships that could be construed as a potential conflict of interest.

Copyright (C) 2019 Thomson and Lamont. This is an open-access article distributed under the terms of the Creative Commons Attribution License (CC BY). The use, distribution or reproduction in other forums is permitted, provided the original author(s) and the copyright owner(s) are credited and that the original publication in this journal is cited, in accordance with accepted academic practice. No use, distribution or reproduction is permitted which does not comply with these terms. 